\title{
Rheumatology meets radiology in the hot soup of Gutta
}

\author{
Tim L Jansen
}

\begin{abstract}
If left untreated, gout may result in radiographic abnormalities, that is, cartilage loss and periarticular osteopenia plus more-or-less gout-specific radiographic abnormalities: spurs, sclerosis, and periostal new bone formation. In the current issue, Dalbeth and colleagues describe findings from about 800 joints in 20 mostly tophaceous patients, which can help clinicians to identify osteopathologic gout: spurs, osteosclerosis, ankylosis and periostal new bone formation, all symptoms of advanced, untreated gout. These are hallmarks of chronic untreated gout and are to be prevented.
\end{abstract}

Gout is the most common inflammatory arthritis in men and an increasingly frequent cause of arthritis in women, and is often not treated adequately [1]; the prevalence of gout is about 2 to $4 \%$ in Europeans and North-Americans. It is a chronic disease based on metabolic urate accumulation and immunologic (hyper)response (Figure 1). Gout is characterized by a spectrum consisting of at least four stages: 1) asymptomatic hyperuricemia, 2) acute arthritic attack, 3) an intercritical period (attack free episodes), and 4) advanced destructive gout (due to urate accumulation and/or acute attacks). During the intercritical period, subjective complaints are lacking but a chronic smoldering inflammation is often present. Chronic inflammatory responses in gout and its sequelae in the locomotor and/ or cardiovascular system are not fully understood, but with modern targeted therapies and translational in vitro research we are starting to unravel these processes. With this information, and if pharmacotherapeutic options are administered appropriately, we, as clinicians, should be able to solve the devilish problems of ancient 'gutta'. Gutta is the origin of the word gout, the Latin word for a

${ }^{*}$ Correspondence: t.jansen@reuma.umcn.nl

Department of Rheumatology, Radboud University Nijmegen Medical Centre, Geert Grooteplein Noord 10, 6525 GA Nijmegen, The Netherlands droplet of devilish fluid, which later turned out to consist of monosodium urate (MSU).

MSU crystal deposition is due to focal hypersaturation and may be associated with reduced blood flow on the one hand, and more acidic and colder microenvironments providing conditions for focal deposition on the other. Changes in the relative proportion of glycosamino- and proteoglycans and lipid concentration add to this process. Both chondroitin sulphate and phosphatidylcholine are constituents of the matrix of joint tissue/ hyaline cartilage and are continuously released into the synovial fluid, where they reflect joint tissue metabolism. MSU crystals can be found in cartilage, tendon sheaths, synovial fluid and subcutaneous tissue. The earliest articular changes result from a coating type of deposition where MSU crystals spread on to and over the cartilage surface as if coated with 'icing sugar' (Figure 2).

Gouty tophi consist of foreign body granulomas composed of mono- and multinucleated macrophages surrounding deposited MSU crystals: the central zone bears MSU crystals, and is surrounded by the corona, a cellular zone of macrophages, mast cells, and plasma cells. The corona is surrounded by a fibrovascular zone. In bone these deposits will result in erosive and destructive disease due to the granulomatous response, which as a process is similar in gout, sarcoidosis and tuberculosis. Tophaceous bone erosions are mediated via the receptor for activation of nuclear factor kappa B (RANK) and RANK ligand (RANKL) pathway [2,3]. Gout arthritis results in IL-1 beta production, which has been implicated to play a pivotal role in bone destruction. Blocking the IL-1 pathway may result in uncoupling of erosiveness in tophaceous disease.

The overhanging appearance of erosions in gout is due to the internal growth process of tophi - that is, they grow inside the erosion. Blocking essential molecules of osteoclastogenesis will prevent the formation of erosions, despite the presence of synovial inflammation. Where osteoclasts are involved in bone erosions via RANKL and IL-1, macrophages are implicated in erosion of unmineralised cartilage via matrix metalloproteinases (MMPs). Recruitment of macrophages into the tophus occurs continuously, and this is the motor for production 


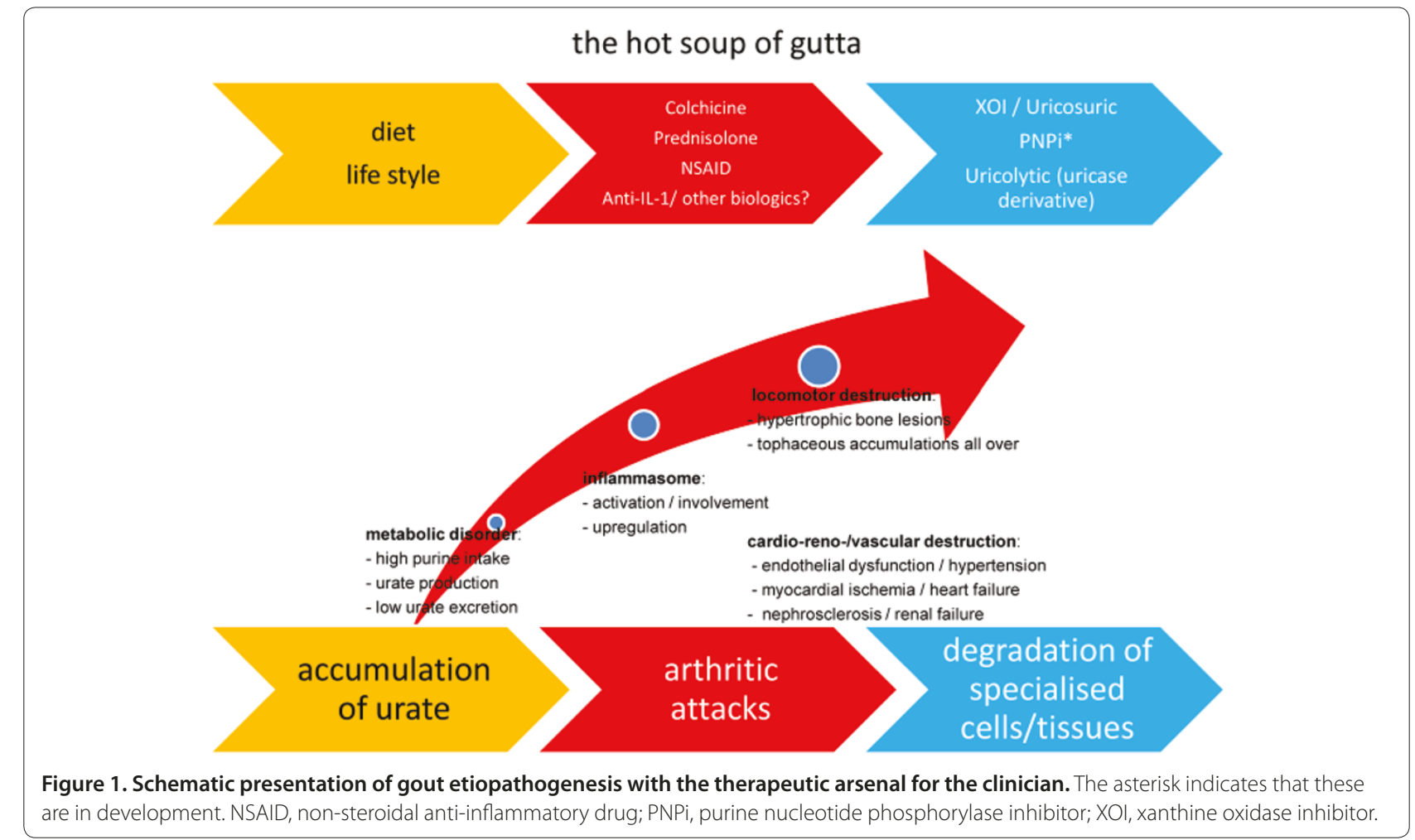

of the proinflammatory cytokine TNF and the TNFinducible lytic enzymes MMP-2 and MMP-9. MSU crystals reduce the effects of anabolic osteoblasts, and seem to alter the normal balance between RANKL and osteoprotogerin, thereby indirectly promoting osteoclastogenesis in MSU-induced arthritis. IL-1 is a key molecule in TNF- and RANKL-induced osteoclastogenesis. The effects of the IL-1 receptor antagonist on markers of bone erosion and cartilage damage appear to be superior to its effects on inflammation, at least in rheumatoid arthritis.

Early detection and treatment of arthritic gout attacks have been prioritized over the past decades. Polarisation microscopy of the punctate (gold standard) and ultrasonography significantly contribute to early diagnosis in gout. Common radiology contributes to late gout diagnoses as it often takes years to develop radiographic abnormalities. In longstanding, often non-arthritic gout, tophaceous bulky disease may even coincide with common as well as more specific radiographic lesions. Dalbeth and colleagues [4] performed a quantitative analysis of radiography and tomography (798 joints photographed) in longstanding, often (80\%) tophaceous gout (20 patients with about 20 years of exposure to a serum urate of about $0.49 \mathrm{mmol} / \mathrm{l}$ ), an interesting experimental setting indeed.

Gout attacks are considered to result in soft tissue swelling, cartilage loss and periarticular osteopenia.
Characteristic but not diagnostic for gout are radiographic features such as punched-out lytic lesions of articular bone [4], not to be confused with rheumatoid erosions. In gout these lytic lesions appear as sharp, often marginated lucent pockets, or discrete subchondral 'pseudocysts' seemingly not connected to the joint. Sclerotic margins signal bone repair in temporarily nonusurating foci. At the periphery of this 'erosive' lesion the bone margin appears to even hang over the tophaceous debris, particularly in hand/foot radiographs [5]. In the presented series about $30 \%$ of small hand joints were 'erosive' at radiography, and a similar percentage at tomography. These 'erosive' lesions differ from rheumatoid arthritis with respect to appearance and the high frequency of occurrence in gout cases. Displacement of the margin probably reflects a gradual increase in size of the urate nidus, growing by multicentric deposition of urate crystals. In the vicinity of tophi, osteoblast activity is reduced and osteoclast activity enhanced. MSU crystals result in cell activation, cytokine production and proteases, all of which enhance focal erosiveness, resulting in bone fragility [6].

If left untreated, new bone formation occurs in joints affected by gout [4]. MSU accumulation near a joint is associated with spurs, periostal new bone formation, ankylosis, and particularly osteosclerosis and osteophytosis. Tomography might be assumed to be more sensitive in detecting radiographic lesions than common 


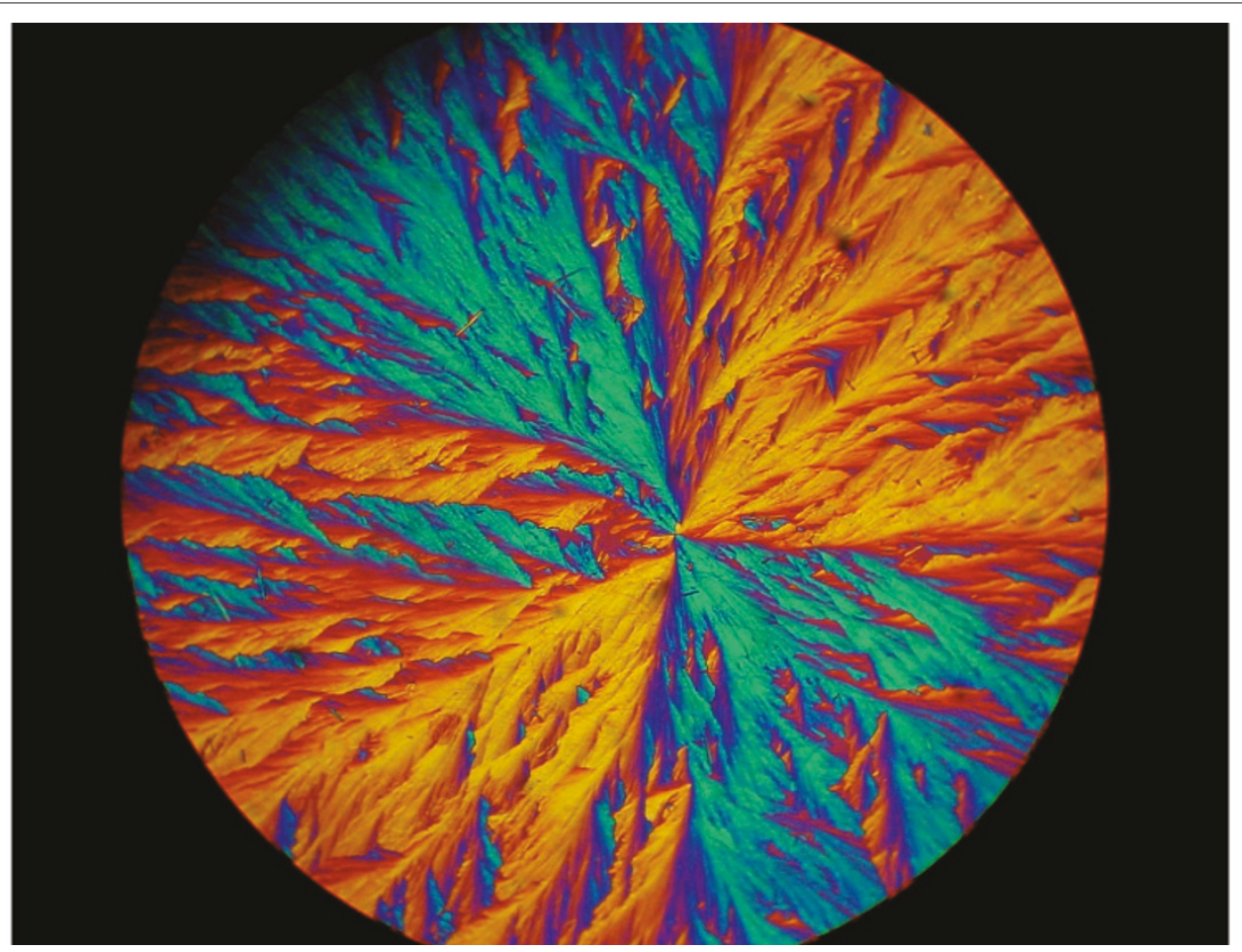

Figure 2. Polarized light microscopy of a fine layer of monosodium urate on a glass slide (with some solitary birefringent needles); magnification $400 \times$.

Table 1. challenges for imaging in gout

\begin{tabular}{|c|c|c|}
\hline Technique & 'Specific' feature & Clinical potential \\
\hline \multirow[t]{5}{*}{ X-ray } & Ankylosis & Daily practice \\
\hline & Osteosclerosis & Long-term follow-up \\
\hline & Osteophyte & In epidemiology \\
\hline & Periostal new bone & In pathophysiology \\
\hline & Spur & In intervention study \\
\hline \multicolumn{3}{|l|}{$C T$} \\
\hline \multirow[t]{2}{*}{ DECT } & Tophaceous load & Experimental application \\
\hline & & In urate lowering therapies demonstrating efficacy \\
\hline \multirow[t]{2}{*}{ HRCT } & Erosion-tophus interrelation & Experimental application/sporadic in daily practice \\
\hline & & In pathophysiology \\
\hline \multirow[t]{2}{*}{ US } & DCS/snow storm & Common use in daily practice \\
\hline & Power Doppler sign & $\begin{array}{l}\text { In diagnostic algorithm for early diagnosis (and possibly follow-up); measuring active } \\
\text { inflammation }\end{array}$ \\
\hline \multirow[t]{2}{*}{ MRI } & Bone oedema/osteitis & Experimental application/daily practice \\
\hline & & In follow-up intervention study; measuring active inflammation \\
\hline
\end{tabular}

$C T$, computerized tomography; $D C S$, double contour sign; $D E C T$, dual energy $C T ; H R C T$, high resolution $C T ; M R I$, magnetic resonance imaging; $U S$, ultrasound.

radiology, and indeed was shown to be twice as sensitive at detecting spurs and 1.5-fold more sensitive at detecting osteophytes [4]. Intraosseous MSU accumulation is correlated with spurs and sclerosis and only weakly correlated with ankylosis and periostal new bone formation. The study by Dalbeth and colleagues [4] does not show data on the (ir)reversibility or pathophysiology of bone erosion in gout, still important unanswered clinical questions. Where exactly do IL-1, TNF, MMP and RANKL fit in, and can therapeutic modulation with anti-IL-1, 
anti-TNF, anti-RANKL and anti-MMP inhibit progression in gout.

Clearly, imaging techniques have exciting new applications [7] (Table 1): dual energy CT reveals later features of gout (that is, tophaceous load), high resolution CT the interrelation between bone erosions and tophi, MRI bone marrow edema/osteitis/tophi/synovitis, and ultrasound early abnormalities, such as snow storm (leukocytes/ urate needles in solution) and double contour sign (a fine layer like icing sugar over the cartilage; Figure 2), and power Doppler can semiquantitatively measure active inflammation.

As bone is a living tissue one would hypothesize that many, if not all, bone changes can be repaired, contrary to cartilage loss. Then, what therapy in these late gout stages is most appropriate? In both early and advanced stages of disease top priority remains urate-lowering therapy to reduce the bodily urate burden; in advanced stages one may theoretically consider osteoclast-targeted therapy (anti-IL-1 or anti-RANKL) to lower osteoclastic bone resorption [8], or T-cell-modulating therapy to reduce stromal infiltrates of $\mathrm{T}$ cells producing RANKL [9]. The most expedient therapy up to now is early starting treat-to-target urate-lowering therapy with allopurinol (and/or uricosuric) in order to prevent all the late, radiological features Dalbeth and colleagues describe.

\section{Abbreviations}

$C T$, computerized tomography; IL, interleukin; MMP, matrix metalloproteinase; MRI, magnetic resonance imaging; MSU, monosodium urate; RANK, receptor

for activation of nuclear factor kappa B; RANKL, RANK ligand; TNF, tumou

necrosis factor.

\section{Competing interests}

The author declares that he has no competing interests.

Published: 19 December 2012

\section{References}

1. Doherty M, Jansen TL, Nuki G, Pascual E, Perez-Ruiz F, Punzi L, So AK, Bardin T: Gout: why is this curable disease so seldom cured? Ann Rheum Dis 2012, 71:1765-1770.

2. Schlesinger $N$, Thiele RG: The pathogenesis of bone erosions in gouty arthritis. Ann Rheum Dis 2010, 69:1907-1912

3. Dalbeth N, Clark B, Gregory K, Gamble G, Sheehan T, Doyle A, McQueen FM: Mechanisms of bone erosion in gout: a quantitative analysis using plain radiography and computed tomography. Ann Rheum Dis 2009, 68:1290-1295.

4. Dalbeth N, Milligan A, Doyle AJ, Clark B, McQueen FM: Characterisation of new bone formation in gout: a quantitative site-by-site analysis using plain radiography and computed tomography. Arthritis Res Ther 2012, 14:R165.

5. Martel W: The overhanging margin of bone: a röntgenologic manifestation of gout. Radiology 1968, 91:755-756.

6. Nguyen $C$, Ea HK, Palazzo E, Lioté F: Tophaceous gout: an unusual cause of multiple fractures. Scand J Rheumato/ 2010, 39:93-96.

7. McQueen FM, Doyle A, Dalbeth N: Imaging in gout - what can we learn from MRI, CT, DECT and US? Arthritis Res Ther 2011, 13:246.

8. Dalbeth N, Smith T, Nicolson B, Clark B, Callon K, Naot D, Haskard DO, McQueen FM, Reid IR, Cornish J: Enhanced osteoclastogenesis in patients with tophaceous gout: urate crystals promote osteoclast development through interactions with stromal cells. Arthritis Rheum 2008, 58:1854-1865.

9. Lee SJ, Nam KI, Jin HM, Cho YN, Lee SE, Kim TJ, Lee SS, Kee SJ, Lee KB, Kim N, Park YW: Bone destruction by receptor activator of nuclear factor kappa beta ligand-expressing T cells in chronic gouty arthritis. Arthritis Res Ther 2011, 13:R164

doi:10.1186/ar4068

Cite this article as: Jansen TL: Rheumatology meets radiology in the hot soup of Gutta. Arthritis Research \& Therapy 2012, 14:126. 